\title{
Supervised Learning Methods and Applications in Medical Research
}

\author{
${ }^{1}$ Yung Ming and ${ }^{2}$ Lily Yuan \\ 1,2 Computing, Peking University, Beijing, China, 100871. \\ 1'ming3233@pku.edu.cn
}

\section{Article Info}

Journal of Computing and Natural Science (http://anapub.co.ke/journals/jcns/jcns.html)

Doi: https://doi.org/10.53759/181X/JCNS202202005

Received 26 May 2021; Revised form 29 June 2021; Accepted 30 September 2021.

Available online 05 January 2022.

(C2022 Published by AnaPub Publications.

\begin{abstract}
Machine Learning (ML) and Artificial Intelligence (AI) methods are transforming many commercial and academic areas, including feature extraction, autonomous driving, computational linguistics, and voice recognition. These new technologies are now having a significant effect in radiography, forensics, and many other areas where the accessibility of automated systems may improve the precision and repeatability of essential job performance. In this systematic review, we begin by providing a short overview of the different methods that are currently being developed, with a particular emphasis on those utilized in biomedical studies.
\end{abstract}

Keywords - Machine Learning (ML, Artificial Intelligence (AI), Supervised Learning.

\section{INTRODUCTION}

Artificial intelligence (AI), particularly machine learning (ML) techniques, has experienced a significant rise in usage over the past decade for a variety of applications. Personal assistants capable of understanding spoken natural language and doing basic activities like gathering data from a calendar, controlling home appliances, and making online purchases, for example, are already being used on thousands of cellphones. The self-driving vehicle is a noteworthy example of cuttingedge AI, since it uses machine vision as well as other sensors to detect its surroundings, as well as automatic monitoring systems to make choices and drive without human intervention.

While the terms AI and ML are often used interchangeably in the general press, ML is just one branch of AI that deals with ways to provide a machine the capacity to "train," that is, to enhance efficiency in particular tasks depending on prior experience or data given [1]. Even though other AI areas such as insight in understanding, algorithms, and optimization computation have had a huge effect on scientific and technological, ML is probably the most intriguing and promising area of AI for medical and scientific applications (Fig 1).

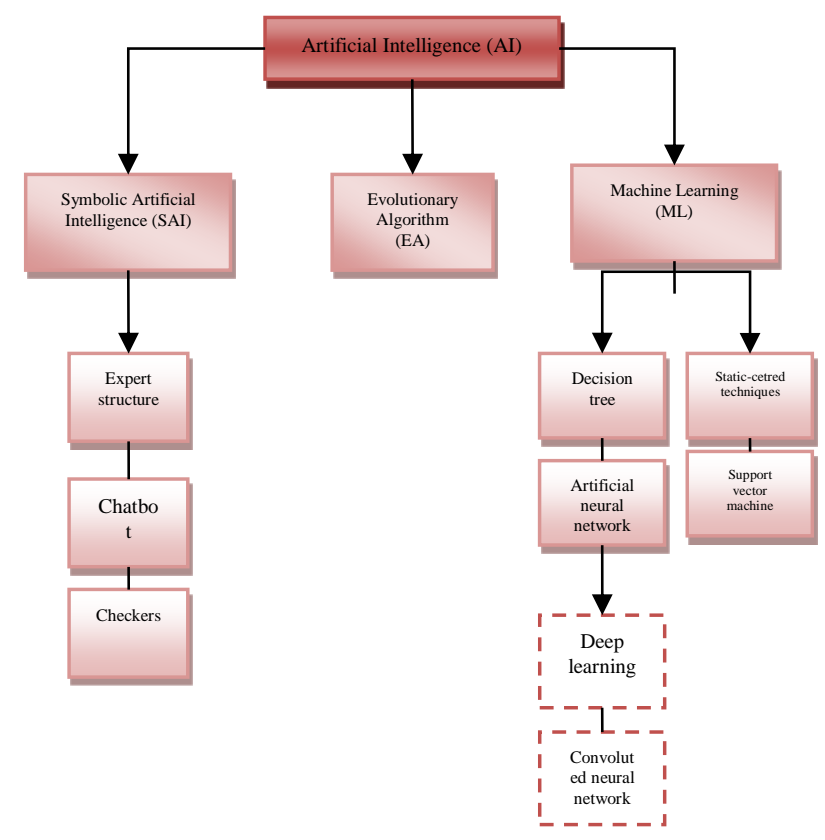

Fig 1. A schematic presentation of the major ML and AI branches with major implication on medical research. 
The presence of data is utilized to teach the machine to execute the required tasks in ML. Because of its nature, machine learning lends itself towards situations wherein inputs is utilized to produce an output depends on certain characteristics of the inputs itself, such as picture categorization. Indeed, image processing is a study field where ML has made significant progress in recent years. A convolutional neural network accomplished super - human achievement in a well-known image processing competition, the ImageNet Large Scale Visual Recognition Contest, for maybe the first time in 2015 could now accomplish functions such as image processing, object recognition (e.g., face recognition system), and monument subtitling better than professional human contractors [2]. Whilst the use of such advanced devices in computed tomography is still in its early stages, specialists generally believe that ML is a genuinely disruptive technology that has the potential to profoundly change how imaging data is processed and used for therapeutic interventions and follow-up. So far, the effect of ML and AI on other fundamental medical research areas has been less noticeable; however, many new applications, such as motion control and mechanical characterisation of tissues, are beginning to emerge.

As shown by the rapid spike in the amount of scientific publications in recent times [3], ML and AI are increasingly being utilized to study clinical problems, particularly in radiology, as well as in other areas such as treatment outcome prediction. In many applications, the presented findings are either promising or already outperform the prior state of the art; for example, ML methods now allow for precise and completely reproducible grading diagnostic spinal degenerative discs on magnetic resonance imaging (MRI) images. Indeed, the present rate of technological advancement is likely to produce additional advantages in the future [4].

With this descriptive review of the literature, we want to raise awareness of AI's present accomplishments and future clinical-related implications in the medical scientific establishment, as well as readers working in other disciplines who are unfamiliar with the technical elements of such technologies. To that end, the article first provides a basic review of AI, with a focus on ML and recent developments that have a practical or prospective effect on clinical research. The following sections describe the current state of the art in use of AI and MLin health sciences, including diagnoses diagnostic practice, future therapeutic prognostication, health information systems, retrieval of information, physiological analysis and categorisation of biological materials, and motion tracking. The Section II below presents a literature survey of AI and ML and its applications. Section III focuses on a critical analysis of the paper while Section IV concludes the paper.

\section{LITERATURE REVIEW}

In [5], authors argue that the earliest steps towards AI may be traced back to the invention of overall processors during WWII, which were accessible for civil usage in the 1950s. The newly accessible computer power enabled the development of symbolic AI systems, which are programs that use a system of regulations to mimic thinking and make choices. Notable systems include those targeted at squares and games of chess, which reached successful performance in the 1970s, and also the earliest chatbots that could mimic a speech recognition discussion to some degree. Similarly, taking advantage of new breakthroughs in neurological research that demonstrated that the autonomic nervous system is made up of a wide network of units conversing via digital currents, investigators began creating so-called artificial neural networks (ANNs), - i.e. channels of neurones emulating the structure of the brain (see Fig 2a), using analog processes.

In [6] authors argue that certain systems, such as the autoencoder, have been demonstrated to be capable of performing basic logical tasks and recognizing categories of patterns, albeit with severe restrictions. Following the first decades of study, there had been a sequence of stages of overall incredulity ("AI winters"), owing to an undervaluation of the sophistication of the issues to be resolved and an absence of adequate computational capabilities, and idealistic phases with additional funding and technology advancements. In the 1980s, expert systems, or computer programs that can solve practical issues using a set of rules drawn from human professional expertise, were effectively used in a variety of academic and industrial areas. ANNs were revitalized in the same years by the discovery of classifier, a strong training algorithm that is still the foundation for their usage today.

In [7] researchers argue that over the past two decades, developments in computer performance and usability, even for tiny research institutions, enabled by graphics processing units (GPUs) with massive parallel computational capacity, have fueled the development of AI technologies for a wide range of practical applications. While intelligent Machines, that is, a computerized programme with a versatile cognition that can do any job possible for humans, is still a long way off, narrow AI, - i.e., a technology that can apply AI exclusively to a particular issue, has achieved extensive usage. Web search engines and voice recognition software are excellent examples of recent technologies' immense potential.

In [8], authors posit that Deep learning is among the sections of AI that is progressing the fastest (see Fig 2b). This Modeling technique, which is transforming study areas like as image analysis, voice recognition, and language processing, is dependent on deep learning models in most deployments, that is, network topologies with many layers. A main driver for the development of deep learning, in associated with improved computational capabilities, was the accessibility of large datasets, or humongous sets of data gathered from different sources, such as the World wide web and medical center (e.g., visualization database systems), which seem to be highly valuable for the efficacious extraction of pattern recognition in practical uses.

In reality, deep learning is used in the majority of the scientific publications that apply AI to medical trials and are mentioned in the sentence "Areas of Application and AI and ML in Medical Research." Section III critically evaluates the aspect of supervised machine learning techniques and application. 


\section{CRITICAL ANALYSIS}

\section{Machine Learning $(M L)$}

The terminology "Machine Learning" was proposed by Arthur Samuel in 1959 to illustrate an area of research that allows machines to understand without having to be expressly configured [9]. This paragraph highlights the key principles of machine learning, which are discussed in more depth elsewhere. The overall goal of machine learning is to generate a prognosis, or to estimate the amount of an expected outcome given an entry, using just characteristics supplied by the model creator or automatically acquired from training data. The following are some examples of popular Machine learning algorithms:

\section{Categorization}

The input is divided into two or more different groups.

The computerized diagnosis based on histological pictures is an example of classification model, in which the computer must determine if an image has characteristics (e.g., textural and colour data) that represent a pathological state. The mechanization of Pfirrmann rating for degenerative discs is an example of a multiclass classifier, whereby an MRI scan of a disc must be classified into one of five categories, range of 1 (normal disc) to 5 (degenerative disc) (severe disc degeneration). Segmentation process, whereby each pixel is labelled according to whether it belongs to a particular area or structure of the brain, is also a kind of classifier. Regression occurs when a task's return is continuously instead of discontinuous [10]. The identification of the dimensions of an architectural landmark in a radiography picture is an illustration of a recurrence issue.

\section{Clustering}

Depending on characteristics learnt from the inputs, the given inputs are sorted into groups. When no a prerequisite understanding of the data's membership to a particular class is provided, cluster analysis is performed to categorize it. Segmentation has been used to classify patients with osteoporotic vertebral fractures into groups depending on pain development, for instance. Another approach to categorize the various types of ML is to consider the nature of the jobs to be completed:

(a) Supervised learning: the machine is trained to determine the outcome from a set of inputs for which the true outcome (ground truth) is established. In most cases, supervised learning entails reducing the value of an error function that explains the distance between both the computer forecasts and the classification algorithm in order to find the best way to translate the input to the output. In clinical research, it is the most frequent type of learning.

(b) Unsupervised learning: the computer learns from data that does not have any contextual information. This kind of learning learns assignment patterns and regularities and characteristics in the inputs in order to extract new knowledge from big data. Clustering is an example of unsupervised learning in action.

(c) Reinforcement learning: rather than having ground truth data accessible at the start of the assignment, input on the accuracy of the implementation is given after the task is finished, serving as a reward or penalty. Reinforcement learning is most often employed in variable or dynamic settings, like as games [11]. Healthcare decision is becoming more popular as a field of applications. Reinforcement learning models are useful for studying how non-human living creatures learn the causality of activities and tasks.

Regardless of the tasks at hand, having big datasets to write code and evaluate its efficiency is key to a good application of machine learning. This need presents significant difficulties in terms of data confidentiality, ethics, legislation, and liability, particularly in medical study.

\section{The Methods of Supervised Learning}

The techniques for supervised methods, which play a critical role among some of the ML activities in clinical research and are discussed in full elsewhere, are briefly summarized in the following sections. The idea of supervised learning is based on the estimate of a function that maps an integer number to an actual output, which may be a picture or a compilation of clinical data about a patient. As a result, the classification model consists of a collection of pairs that include inputs and a known comparative output.

After determining the mapping function, it may be used to analyze added information for which the output value is not known [12]. If there are enough training examples and a suitable learning method is used, the algorithm will be able of generalization successfully, or give correct results for inputs that are similar but not identical to those in the dataset. Alternatively, the forecasts may expose fitting problem, which occurs when the adaptive learning is indeed not inherently advanced to encapsulate the representation of the input documentation but is unable to build a predictive model on additional information, or regression problems, which occurs when the adaptive learning is indeed not sufficiently advanced to encapsulate the representation of the input records but is unable to build a predictive model on extra information. 


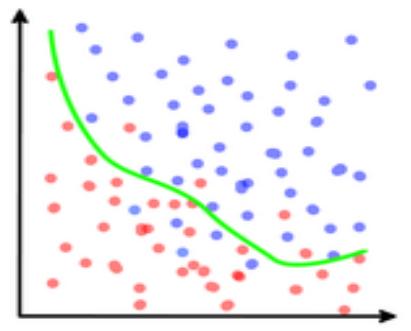

Fig 2c: Conceivable fitting

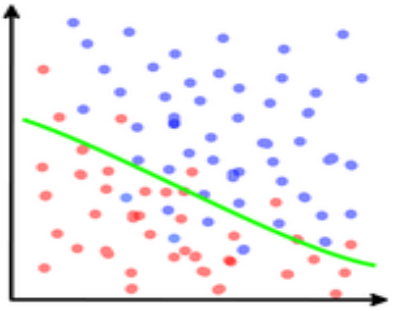

Fig 2b: Underfitting

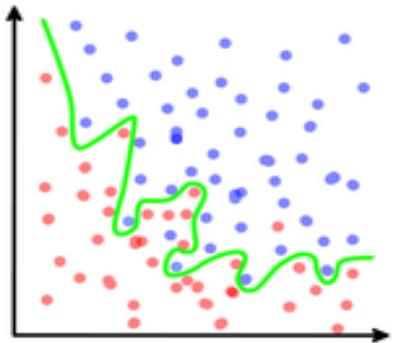

Fig 2c: Overffiting

Fig 2 (a, b, and c). Sample of conceivable fitting, underfitting and overfitting with the task categorization of the binary class.

\section{Methods Based On Statistics}

Although it may seem paradoxical to examine regression analysis in the context of machine learning, it is an excellent example of a basic technique for creating a function that translates an input (a integer, or more often a vectors of integers) to an output. In fact, every kind of input may be mathematically expressed as a variable vector of integers, often referred to as features, and then analyzed using linear regression. Parameters are a collection of variables that describe data and may be basic and human accessible (for example, a patient's age and sex) or more complex to understand (for example, picture characteristics extracted using specialized algorithms such as ORB and SIFT, or texture and form analysis). A picture, such as a radiograph, may be regarded as an array of integer integers with length equivalent to the total of pixel in the picture; each component of the array will include the color (gray value) of the particular pixel even without extracting features. The use of regression analysis, especially in the situation of complicated and huge inputs, is simple from this viewpoint.

The least square technique is frequently used to fit the linear regression function, and therefore serves as the training procedure. In this instance, reducing the Mean Square Error (MSE) in between forecasts and the inputs equates to completing a linear interpolation; MSE thus reflects the algorithm's gradient descent. MSE is also known as L2 loss in the ML literature, while the L1 losses are indeed the Mean Absolute Error (MAE), which seems to be a viable option for regression issues [13]. Regression analysis is susceptible to misclassification because to its minimalism and incapacity to capture non - linear behavior. As a result, it is not the technique of choice for complicated ML classification or regression.

For classification issues, regression analysis is the comparable of regression analysis. The sigmoidal function, a non linear curve that indicates the likelihood that an inputs is translated to the "1" outputs, is used to fit the outputs (one or many continuous values) to a different configuration ( 0 or 1$)$ in its simplest form. A "1" is anticipated if the output frequency is greater than or equal to 0.5 , while a " 0 " is projected if the output possibility is less than or equal to 0.5 . Logistic regression may be successfully extended to multiclass categorization issues in addition to forecasting binary outcomes. MSE is not the best option for acting as a loss function in logistic regression; instead, specialized functions like cross entropy are used. For the most ML classifiers, logistic regression, like linear regression, is surpassed by more advanced algorithms.

The Classification algorithm, which is based on Bayes' principle of probabilistic reasoning, is another technique originating from parameter estimation that has made its way into the ML literature. The naive Bayes classification, which assumes that features are independent of one another, is particularly easy to build, quick to train even for extremely large trained data, and theoretically highly successful in applications where component neutrality is plausible. Bayes classifier is used in medical tests to classify osteoporotic fractures and for CAD. 
Vector Support Machines for Vectors

Conducting classifications assignment refers to finding a division of the space that separates the spots pertaining to the different classes, taking each input from the dataset as a multivariate vectors and thus as a pixel in a subspace. A support vector machine (SVM) is a method that creates a support vectors, or a set of hyperplanes, that divides space in such a way that the vertices of various classes are efficiently and ideally segregated (Fig 3).

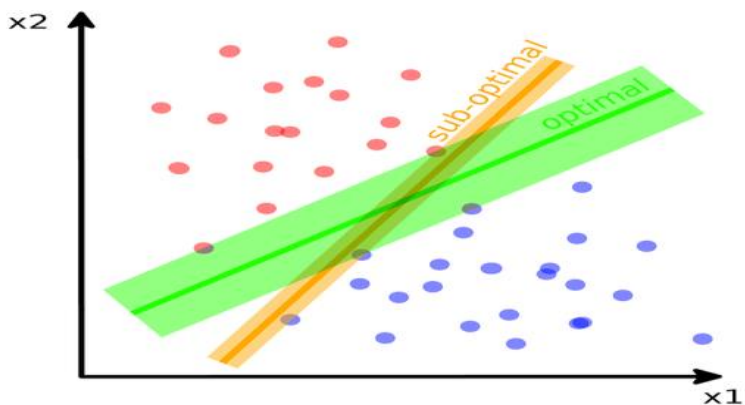

Fig 3. A basic support vector machine (SVM) for classifier

In a nutshell, the SVM creates the best higher dimensional space (in green) between the two classifications, increasing the distance between them. A non-optimal descriptor (orange) is also presented, which divides the two classes properly but with a lesser separation. In the higher dimensional space, the SVM works (x1 and $\mathrm{x} 2$ in the exemplary images). SVMs are effective tools for classifiers linear classifiers such as picture fragmentation. Despite the fact that SVMs were first published in 1963 [14], they are still extensively used today and may outperformed more modern methods in some situations, such as when the supervised learning is restricted in size. SVMs have been utilized in clinical research for evaluating disc deterioration and categorization of scoliosis curvature subtypes, for instance. SVMs may be used to solve issues like nonlinear regression and classification, and unsupervised classification (e.g., clustering).

\section{Regression and Classification Decision Trees}

The usage of tree-like patterns in AI may be traced back to Arthur Samuel's pioneering checkerboard systems. The clustering algorithm was originally used in the 1950s for regression and classification. Decision tree algorithms are now widely used in a variety of areas, including commerce and the military. Notably, they are often employed in medical services to determine the most suitable medical therapy. A classification and regression decision tree (CART) in machine learning connects the value of variables to the potential results, thus performing a predictive data mining job via a set of parameters. The tree divides into parts for each condition, with terminals nodes indicating the decision's result; because of its unique form, CARTs are simpler to comprehend for users than other ML methods.

CARTs may be trained using specialized methods based on huge amounts of input data, which are usually not computationally expensive and therefore appropriate for extremely big datasets. CARTs are susceptible to imbalanced datasets, which may be mitigated by using methods like pruning, which lowers the scale of the tree, and regression trees, which combine several decision trees randomized selections of the characteristics and mean their forecasts. CARTs and regression trees have indeed been utilized in biomedical studies for a variety of purposes. Decision tree algorithms have been utilized as a healthcare delivery system for the treatment of back pain and the postoperative screening of individuals with adult cervical spondylosis. Other uses include forecasting proximal junctional failure and assessing the main fixation strengths of spinal screw (Fig 4).

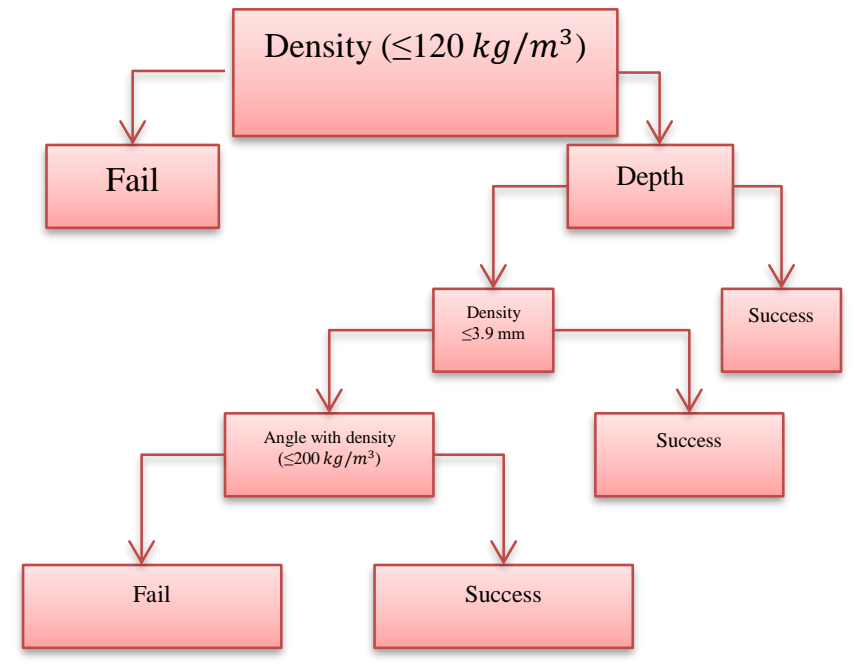

Fig 4. Sample of the decision tree to predict the potential risks of failure of the spinal screw 
Artificial Neural Networks (ANNs)

ANNs are the branch of machine learning that has experienced the most significant advancements in recent decades, to the point where the public at large has mistakenly associated it with machine learning. There are numerous applications of ANNs in healthcare study and therapeutic chemistry, which are detailed in the phrase "Implementations of AI and ML in biomedical studies."

ANNs are scientifically influenced systems that are based on how neurotransmitters in the mind are linked and respond. Information circulates from the input variables to the output values through interconnected neurons that are arranged in strands and perform basic processes like making configuration of their input variables magnified by a mass, and then manufacturing the outcome through a sequential or variational stimulation feature, imitating the guidelines of Hebbian teaching (see Fig 2c). Normalization parameters may be included in the systems to reduce the danger of collinearity by compensating significant weight levels via a punishment variable. The method of teaching an ANN entails determining the best set of weights such that the contributions from the test dataset are analyzed and passed through the tiers, culminating in outcomes that closely resemble the true data.

MSE, MAE, and cross entropy, which are the same cost measures discussed in the preceding sections, are extensively used to educate ANNs and as success indicators. In its most basic form, the deep residual practice technique involves calculating the variations of the loss signal with respect to each load capacity and then modifying the individual weight by the magnitude of the corresponding divergence magnified by a factor, the teaching pace. Initialising the procedure causes the logistic regression to shrink, eventually reaching a limit after divergence is attained. More complex approaches, such as back - propagation algorithm and Adam, have largely replaced this vertical reduction technique in terms of achieving faster and more reliable completion.

ANNs are utilized to solve regression and classification challenges in a variety of industries and academic disciplines. Whereas learning algorithms are the most common implementation of ANNs in medical trials, these models can also be used for uncontrolled activities and reinforced training [15]. Network capacity frameworks that are streamlined to consider specific issues have been established based on previous case studies such as the single sheet classifier, a simple regression classifier model consisting of a single line of output signals tied directly to the input data through a sequence of barbells. For instance, generated algorithms are increasingly commonly used to produce new information that matches some properties with existing data, while recursive neural networks are commonly used to analyse data while remembering past entries. Voice recognition and computerized translation are two applications of the latter technologies.

\section{Applications of AI and ML in Medical Research}

Artificial intelligence (AI) is proving a significant influence in various clinical research domains, and this effect is projected to grow in the years. We outline the reported uses of AI and ML in several sectors of medical research, such as medical testing, medical result forecasting, and choice assistance technologies, in the following lines. Biophysics and motion detection are examples of applications that are more intimately tied to scientific knowledge.

\section{Localization and Labeling of Spinal Structures}

From radiographic images such as conventional radiography, computed tomography (CT), and MRI scans, machine learning techniques have been used to obtain information such as the position of vertebral, disc, and vertebral morphology. In fact, locating anatomical landmarks in an imagery database is often the first step in developing completely automated systems for detecting and classifying diseased characteristics, as well as predicting the fate of treatment.

For localized problems, appropriate ML approaches have been utilized in conjunction with strategies not technically connected to ML, such as binarization and intuitive exploration. Schmidt used a classification algorithm to obtain a correlation outline of each intervertebral centroid's position in MRI scans, which was then used by probably - based graphs framework to deduce the most suitable place, resulting in a total fault classification of $6.2 \mathrm{~mm}$ when compared to a sentient comparison. A pattern classifier is used, the prismatic distribution of directed slopes, to educate an SVM for discs navigation, with average translation defects varying between 2.6 and $3.6 \mathrm{~mm}$ depending on the discs elevation. The procedure was rooted on a sequence, which is a rectangle region that deflects over a mega weighted copy of the classic portrait; the benefit of the source image is estimated for each role of the display, and transferred as feedback to the SVM to ascertain if the existing refers to the structural a vertebral column disc.

A graphic approach is utilized to estimate the placement of each particular disc once a collection of the most probable disc positions has been established. The procedure was broadened and modified by the same researchers to allow for the localization of the vertebral, with normal defects of less than $4 \mathrm{~mm}$. ANNs and learning techniques have lately been used for the localisation of spine components. Hybrid technique is employed that included a decision tree algorithm that performed a first basic specificity before driving a deep CNN; the overall localized uncertainties for the center of the vertebral discs were 1.6 to $2 \mathrm{~mm}$, which was a significant increase over the prior state of the art, which was not reliant on machine learning. CNNs are employed as well, both relying on $2 \mathrm{D}$ combinations (considering individual slices independently) and a unique 3D convolution level. Convolution operation is employed to locate the intervertebral clusters using a six-layer computational model: the system selected the vectors relating each voxel in the sample to the midpoint.

The results were then utilized to calculate a mathematical estimation of the intervertebral centroid's most likely position. Payer offered an alternate approach, in which they employed 2D and 3D CNNs to create prediction base maps of landmarks positions; nonetheless, the technique was not deployed to imaging data. The identification process was conducted in various articles by applying a modeling method once a good placement of the intervertebral or discs centroids 
had been obtained. Latest research has attained significant precision with complicated models capable of performing feature and intervertebral clustering placement using the entire 3D information as input, without using any rough location or rolling frame techniques. In CT scans of people who suffer from diverse diseases as well as those exposed to therapeutic machinery, Yang were able to attain localized problems for the intervertebral center between 6.9 and 9 mm, with widely varying depth of field and picture quality. In fact, cutting-edge systems for locating and classifying spine components have outperformed skilled human viewers. Business Image and Data storage Communication Systems and widely accessible medical image processors now include identification and labelling features, while technical specifics have not been made public.

\section{Segmentation}

Interpreting the article's information, or segregating the visual into areas at the low resolution so that each pixel corresponds to a specified zone, is a major challenge in image recognition. Semantic categorization is the name given to this procedure, which can be done manual or automatic. Because it is so important in applications like machine learning and automated vehicles, there has been a lot of research done on it. A classification model in radiography should typically establish which particular example an image contains to, in addition to evaluating if it relates to a disc, for instance (e.g., either L1 to L2 or L2 to L3). This kind of division is referred to as semantic segmentation, and it is the most useful in medical investigations.

Measuring the accuracy of a segmentation method requires the development of quantitative measures, which would be less apparent than the information loss used in localisation assignments. Numerous metrics have been proposed in earlier research, and the most popular ones are indeed the Dice similarity coefficient (DSC) quantifies the degree of spatial overlap between both the divided picture and the original data. The mean surface distance (MSD) represents the mean separation in between each interface pixel of the divided ground and the nearest surface pixel inside the actual truth.

Many publications presented approaches for clinical segmentation that did not utilize ML techniques and, in some instances, needed human interaction; completely automated systems were also published. Other techniques depended on matching dynamic physical models to the pictures by way of optimization processes. Among the numerous published approaches, those based on graphs and standardized cuts, as well as methods developed from them, were particularly effective. Scientists, for example, obtained DSC readings of 0.88 or MSD at 2.7 millimeters by utilizing normalized cuts. The posture and form of the objects to be subdivided are parameterized in a myriad of criteria in marginal space learning. A vast range of options spanning the subspace, that is, representing all potential postures of the item, are then developed; the best assumption is chosen using a classifier. CNNs built especially for applications such as categorization have been utilized in recent years.

Researchers utilized a deep CNN comprising 3D neural network to calculate the likelihood of reference to a specific area at the pixel level. To improve the segmentation, postprocessing methods such as binarization and flattening were employed. Researchers used a three - dimensional CNN with a recollection element to recall which vertebrae had previously been categorized. To analyze huge datasets, the method employs a 3D gliding window approach that first identifies the location in which the windows include a complete vertebra before doing pixel-level classification using a deep classification. The memory would then be modified such that if a part of the previously split vertebrae is recognized when searching for the following ones, it is disregarded. We were able to obtain excellent precision with this technique, with a mean DSC near 0.94 as well as an MSD near $0.2 \mathrm{~mm}$.

\section{CONCLUSION}

In conclusion, machine learning and artificial intelligence (AI) are developing disruptive innovation that have already achieved a significant degree of development, allowing them to have a significant effect on a variety of study areas. Due to recent advances in pattern recognition and increased accessibility of supercomputers, such as potent GPUs, machine learning and image recognition are gaining traction. Although the majority of current clinical research studies utilizing AI and ML methods have been focused on medical imaging, a growing effect on other areas such as therapeutic dynamics is anticipated in the near future. Integrity, security and privacy, and the danger of skewed predictions, are all ethical concerns that legislators and regulatory authorities are presently considering. ML has been used for diagnostic reasons since the 1980s.

\section{References}

[1]. "Supervised Learning Classification Of Incomplete Clinical Data", Informatics and Applications, 2017. Doi: 10.14357/19922264170303.

[2]. J. Ali, "Response: Maximising the learning afforded by supervised learning events", The Clinical Teacher, vol. 11, no. 5, pp. 407-407, 2014. Doi: $10.1111 /$ tct.12245.

[3]. "An Insight Into Supervised Learning Using Support Vector Machines", International Journal of Recent Trends in Engineering and Research, vol. 3, no. 8, pp. 84-87, 2017. Doi: 10.23883/ijrter.2017.3391.mezpj.

[4]. "Crop Yield Prediction With Supervised Learning Techniques", Strad Research, vol. 7, no. 7, 2020. Doi: 10.37896/sr7.7/005.

[5]. V. Krajca and S. Petránek, "4. Classification of EEG graphoelements with supervised and unsupervised learning algorithms", Clinical Neurophysiology, vol. 125, no. 5, p. e26, 2014. Doi: 10.1016/j.clinph.2013.12.042.

[6]. S. Gupta, "Performance Evaluation of Supervised Learning Algorithms on Hate Speech Detection", Journal of Advanced Research in Dynamical and Control Systems, vol. 12, no. 7, pp. 2953-2960, 2020. Doi: 10.5373/jardcs/v12sp7/20202440.

[7]. A. Dallal and Z. Mao, "S184. Sparse representation and classification of neural spikes using supervised dictionary learning", Clinical Neurophysiology, vol. 129, pp. e210-e211, 2018. Doi: 10.1016/j.clinph.2018.04.544.

[8]. S. Yadav and V. S., "EEG Classification using Semi Supervised Learning", International Journal of Trend in Scientific Research and Development, vol. -3, no. -3, pp. 1441-1445, 2019. Doi: 10.31142/ijtsrd23355. 
[9]. H. Hirai, K. Murota and M. Rikitoku, "Electric Network Classifiers For Semi-Supervised Learning On Graphs", Journal of the Operations Research Society of Japan, vol. 50, no. 3, pp. 219-232, 2007. Doi: 10.15807/jorsj.50.219.

[10]. P. Athanasopoulos and D. Albright, "A Perceptual Learning Approach to the Whorfian Hypothesis: Supervised Classification of Motion", Language Learning, vol. 66, no. 3, pp. 666-689, 2016. Doi: 10.1111/lang.12180.

[11]. R. Sharma, "Study of Supervised Learning and Unsupervised Learning", International Journal for Research in Applied Science and Engineering Technology, vol. 8, no. 6, pp. 588-593, 2020. Doi: 10.22214/ijraset.2020.6095.

[12]. T. Lee and S. Yoo, "Augmenting Few-Shot Learning With Supervised Contrastive Learning", IEEE Access, vol. 9, pp. 61466-61474, 2021 Doi: $10.1109 /$ access.2021.3074525.

[13]. J. Yih, "Supervised Clustering Algorithm for University Student Learning Algebra", Advanced Materials Research, vol. 542-543, pp. 13761379, 2012. Doi: 10.4028/www.scientific.net/amr.542-543.1376.

[14]. A. Fong and G. Hong, "Boosted Supervised Intensional Learning Supported by Unsupervised Learning", International Journal of Machine Learning and Computing, vol. 11, no. 2, pp. 98-102, 2021. Doi: 10.18178/ijmlc.2021.11.2.1020.

[15]. E. Ngozi Chidozie, "Osteoporosis Risk Predictive Model Using Supervised Machine Learning Algorithms", Science Research, vol. 5, no. 6, p. 78, 2017. Doi: 10.11648/j.sr.20170506.11. 